\title{
Cattle gastrointestinal nematode egg-spiked faecal samples: high recovery rates using the Mini-FLOTAC technique
}

\author{
Alessandra Amadesi ${ }^{*}{ }^{*} \mathbb{D}$, Antonio Bosco ${ }^{1}$, Laura Rinaldi ${ }^{1}$, Giuseppe Cringoli ${ }^{1}$, Edwin Claerebout $^{2}$ \\ and Maria Paola Maurelli ${ }^{1}$
}

\begin{abstract}
Background: Faecal egg count (FEC) techniques are commonly used to detect gastrointestinal nematodes (GINs) in cattle and to determine anthelmintic efficacy/resistance through the faecal egg count reduction test (FECRT). MiniFLOTAC is one of the techniques recommended for a standardised FEC/FECRT of helminth eggs in cattle. However, only one paper evaluated the recovery rate of GIN eggs by Mini-FLOTAC (compared to McMaster and modifiedWisconsin method) in cattle, using only a level of contamination of 200 eggs per gram (EPG) of faeces and using GIN eggs collected from goat faeces to spike faecal samples from cattle. To further study the recovery rate of GIN eggs from cattle faeces, this study was conducted in two laboratories, one in Belgium and one in Italy to evaluate the sensitivity, accuracy, precision and reproducibility of the Mini-FLOTAC and McMaster techniques (at two reading levels: grids and chambers) for the detection of GIN eggs in spiked bovine faecal samples.
\end{abstract}

Methods: In both countries, spiked cattle faecal samples with five different levels of egg contamination (10, 50, 100, 200 and 500 EPG) of GINs were used. The study was performed in both laboratories by the same expert operator and using the same standard operating procedures (SOPs) for the Mini-FLOTAC and McMaster techniques. Sensitivity, accuracy and precision were calculated for each technique and for each level of contamination. Statistical analyses were performed to evaluate differences in performance between the two techniques.

Results: Mini-FLOTAC had a higher sensitivity (100\% at all EPG levels for Mini-FLOTAC vs 0-66.6\% for McMaster chambers and grids at levels<100 EPG), a higher accuracy (98.1\% mean value for Mini-FLOTAC vs 83.2\% for McMaster grids and $63.8 \%$ for McMaster chambers) and a lower coefficient of variation (10.0\% for Mini-FLOTAC vs $47.5 \%$ for McMaster grids and $69.4 \%$ for McMaster chambers) than McMaster. There was no significant difference in the recovery of GIN eggs between the two studies performed in Belgium and in Italy.

Conclusions: The high GIN egg recovery rate detected by Mini-FLOTAC and the similar results obtained in Belgium and in Italy indicated that the diagnostic performance of a FEC technique was not dependent on the laboratory environment.

Keywords: Gastrointestinal nematodes, Cattle, Faecal Egg Count, Mini-FLOTAC, McMaster, egg-spiking, Belgium, Italy

*Correspondence: alessandra.amadesi@unina.it

1 Department of Veterinary Medicine and Animal Production, University of Naples Federico II, CREMOPAR, Naples, Italy

Full list of author information is available at the end of the article

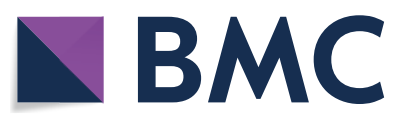

(c) The Author(s) 2020. This article is licensed under a Creative Commons Attribution 4.0 International License, which permits use, sharing, adaptation, distribution and reproduction in any medium or format, as long as you give appropriate credit to the original author(s) and the source, provide a link to the Creative Commons licence, and indicate if changes were made. The images or other third party material in this article are included in the article's Creative Commons licence, unless indicated otherwise in a credit line to the material. If material is not included in the article's Creative Commons licence and your intended use is not permitted by statutory regulation or exceeds the permitted use, you will need to obtain permission directly from the copyright holder. To view a copy of this licence, visit http://creativecommons.org/licenses/by/4.0/. The Creative Commons Public Domain Dedication waiver (http://creativecommons.org/publicdomain/zero/1.0/) applies to the data made available in this article, unless otherwise stated in a credit line to the data. 


\section{Background}

Gastrointestinal nematode (GIN) infections may negatively influence animal health, welfare and productivity in grazing cattle worldwide [1]. The negative impact of GIN on livestock farming is further exacerbated by the emergence of anthelmintic resistance (AR) in cattle nematodes [2-4].

In order to limit AR and the misuse/abuse of anthelmintics in cattle, the use of regular diagnostic testing is suggested as one of the options for a sustainable control strategy [5]. Diagnostic methods for GIN include faecal egg count (FEC) techniques that are commonly used in parasitological research and veterinary practice to indirectly assess GIN burdens and determine anthelmintic efficacy/resistance through the faecal egg count reduction test (FECRT) [6]. FEC techniques based on easy-touse devices with high diagnostic performance in terms of sensitivity, accuracy, precision and reproducibility are suggested to perform reliable and exploitable FEC/ FECRT in cattle $[5,7]$.

Mini-FLOTAC is considered a good candidate for a standardised FEC/FECRT of helminth eggs in livestock [8]. This method, in fact, has been compared with different diagnostic techniques, i.e. Cornell-Wisconsin, McMaster and FECPAK, and was shown to be more sensitive, accurate and precise for FEC and FECRT of GINs in sheep [7, 9-12]. Mini-FLOTAC has been also successfully used to perform FEC and FECRT (in the laboratory and on-farm) in cattle $[5,7,13,14]$. However, only a single study by Paras et al. [7] evaluated the recovery rate of GIN eggs by Mini-FLOTAC (compared to McMaster and modified-Wisconsin) in cattle. The authors found an accuracy of $70.9 \%$, but eggs used to spike samples were collected from goats and only one level of contamination (i.e. 200 eggs per gram of faeces, EPG) was used.

To further investigate the recovery rate of added GIN eggs from cattle, the present paper reports the findings of a study conducted in two laboratories, one in Belgium and one in Italy, to compare Mini-FLOTAC and McMaster (at two reading levels, i.e. grids and chambers) methods, in terms of sensitivity, accuracy, precision and reproducibility, using GIN egg-spiked faecal samples at five different levels of contamination $(10,50,100,200$ and 500 EPG).

\section{Methods}

\section{Study design and sampling}

The study was conducted in two laboratories, one in Belgium and one in Italy.

In Belgium, GIN-positive and negative faecal samples were collected from Belgian Blue cattle stabled at the experimental farm of the Faculty of Veterinary Medicine (Ghent University). Positive samples were collected from calves (6 months-old) experimentally infected with 50,000 third-stage larvae (L3) of Ostertagia ostertagi ( $n=2$ calves) or Cooperia oncophora ( $n=2$ calves), whilst negative samples were collected from uninfected adult ( $>24$ months-old) housed cattle ( $n=2$ calves).

In Italy, GIN positive and negative faecal samples were collected from Podolian adult cattle ( $>24$ months-old) in a commercial farm located in the Salerno Province, Campania region. Positive samples were collected from cattle at pasture, naturally infected by different species of GINs, whilst negative samples were collected from stabled cattle. Each sample was analysed in five replicates by the FLOTAC basic technique [15] with an analytical sensitivity of 1 egg per gram (EPG) of faeces to determine the presence/absence of GIN eggs.

Both in Belgium and in Italy, the positive cattle were used as donors for the extraction of GIN eggs from faeces, using a mass recovery method, i.e. a method that employs 4 sieves of different mesh size $(1 \mathrm{~mm}, 250 \mu \mathrm{m}$, $212 \mu \mathrm{m}$ and $38 \mu \mathrm{m}$ ) in order to separate the eggs from the faeces, as described in Bosco et al. [12]. Eggs were recovered by washing the $38 \mu \mathrm{m}$ sieve with tap water, and centrifuging the eluate for $3 \mathrm{~min}$ at $4000 \times \mathrm{g}$. To concentrate the GIN eggs, the supernatant was removed by a water pump and the pellet was resuspended in $5 \mathrm{ml}$ of a $40 \%$ sucrose solution.

After centrifugation for $3 \mathrm{~min}$ at $4000 \times g$, the supernatant was transferred to a new tube, diluted with an equal volume of tap water and centrifuged again for $3 \mathrm{~min}$ at $4000 \times g$. The supernatant was removed to reduce the final volume of the egg preparation to $5 \mathrm{ml}$. Then, 10 aliquots of $0.1 \mathrm{ml}$ each were taken, after a thorough homogenization of egg preparation into two tubes for 10 times (avoiding foam formation) for each aliquot to provide precise counting of eggs [12]. Finally, the number of eggs was counted at $100 \times$ magnification.

The egg suspensions were added to five confirmed negative faecal samples of $200 \mathrm{~g}$ each to obtain five samples with different EPG levels: 10, 50, 100, 200 and 500 EPG. Each sample was analysed, using saturated sodium chloride solution (specific gravity $=1.200$ ), by two FEC techniques: Mini-FLOTAC [8] and a modified McMaster [16] technique at two reading levels, i.e. grids and chambers. In total, 12 replicates were used for each method and for each EPG level.

From each homogenised faecal sample, for each EPG level, $60 \mathrm{~g}$ were weighed for the Mini-FLOTAC technique $(5 \mathrm{~g}$ for each replicate; dilution ratio $=1: 10$; reading volume $=2 \mathrm{ml}$; analytical sensitivity $=5 \mathrm{EPG}$ ) and $36 \mathrm{~g}$ for the McMaster technique (3 $\mathrm{g}$ for each replicate), reading the two grids (dilution ratio $=1: 15$; reading volume $=0.30$ $\mathrm{ml}$; analytical sensitivity $=50 \mathrm{EPG}$ ) and the two chambers (reading volume $=1 \mathrm{ml}$; analytical sensitivity $=15 \mathrm{EPG}$ ). 
All samples were prepared, analysed and read at $100 \times$ magnification by the same expert operator in Belgium and Italy.

\section{Coprocultures}

In Italy, a pooled faecal culture was performed in order to identify the nematodes to the genus level, following the protocol described in MAFF [16]. Developed third-stage larvae (L3) were identified using the morphological keys proposed by van Wyk \& Mayhew [17]. Identification and percentages of nematodes by genus were conducted on 100 L3; if a sample had 100 or less L3 present, all larvae were identified.

\section{Statistical analysis}

EPG values for each technique and for each GIN infection level were calculated by multiplying the raw counts by the appropriate multiplication factor (e.g. 5 for MiniFLOTAC, 50 for McMaster grids and 15 for McMaster chambers) and then, the mean of the replicate counts for each sample was calculated. The sensitivity of each method was estimated using the following formula: [(total number of positive samples observed/12, i.e. total number of replicate spiked samples performed for each method and for each level of contamination) $\times 100$ ].

To evaluate the precision of each method, a coefficient of variation $(\mathrm{CV})$ [(standard deviation/mean egg count $\times 100$ ] was calculated for each set of replicate counts for each method and level of EPGs. Furthermore, the accuracy of each method was determined by the percentage (\%) of egg recovery calculated for each level of contamination, using the following formula: \% egg recovery $=[($ observed FEC/ true FEC $) \times 100]$. Boxplots (indicating median, percentiles and outliers) were used to show the precision and accuracy of each technique for each of the five levels of egg contamination. The non-parametric Kruskal-Wallis test with Dunn's post-hoc test were used to compare all the observed values to the true FEC value for each technique and for each level of contamination.

Finally, a logistic regression model was developed in order to evaluate the predicted accuracy of each technique. The Mann-Whitney comparison test was used to compare the GIN egg recovery rates by Mini-FLOTAC and McMaster (reproducibility) in the two different laboratories (Belgium and Italy) using different samples from cattle experimentally (Belgium) or naturally infected by different GIN species (Italy), of different ages (calves in Belgium $v s$ adult cattle in Italy) and breed (Belgian Blue vs Podolian).

All statistical analyses were performed in GraphPad Prism v.8 (Graph Pad Software, San Diego, CA, USA). Significance testing was set at $P<0.05$.

\section{Results}

A total of 360 counts were performed. The Mini-FLOTAC technique showed a sensitivity of $100 \%$ at all the EPG levels whilst the McMaster technique (reading either grids or chambers) showed a sensitivity of $100 \%$ only for levels $\geq 100$ EPG. Below 100 EPG the sensitivity of McMaster grids and chambers ranged between $0-66.6 \%$. Figure 1 and Table 1 show the boxplot, the precision (CV \%) and the accuracy (\%) of the observed mean EPG for each country at each level of egg contamination for Mini-FLOTAC, McMaster grids and chambers. The boxplots of the Mini-FLOTAC technique (Fig. 1) were very narrow for each contamination level, thus indicating a high precision compared to the McMaster grids and chambers. CVs for McMaster grids and chambers were higher than those of MiniFLOTAC, especially for low counts.

The Kruskal-Wallis test showed that there were significant differences for McMaster grids and McMaster chambers between observed and true EPG values at 10 EPG (Kruskal-Wallis H-test: $\chi^{2}=57.8, d f=3, P<0.0001$ ), 50 EPG (Kruskal-Wallis H-test: $\chi^{2}=44.5, d f=3$, $P<0.0001), 100$ EPG (Kruskal-Wallis H-test: $\chi^{2}=43.4$, $d f=3, P<0.0001)$ and 200 EPG (Kruskal-Wallis H-test: $\left.X^{2}=17.8, d f=3, \quad P<0.0001\right)$ levels of contamination, whilst at the level of 500 EPG, only McMaster grids (Kruskal-Wallis H-test: $\chi^{2}=20.4, \quad d f=3, \quad P>0.05$ ) showed no statistically significant difference between observed and true values. This finding was confirmed by the results of the logistic regression (Fig. 2), the McMaster grids showing a low predicted accuracy related to low FEC, whilst becoming more accurate only when the FEC level increased, i.e. at 500 EPG.

Finally, the McMaster chambers had a low accuracy at all the levels of contamination, whilst the Mini-FLOTAC did not show any significant difference between the observed and the true values at all the EPG contamination levels.

$P$-values from the Mann-Whitney test ranged from 0.215 to 0.977 (from 10 to 500 EPG levels), showing that there was no significant difference of GIN egg recovery rates (either using Mini-FLOTAC or McMaster) obtained in Belgium and in Italy.

\section{Coprocultures}

The following GIN genera were detected in the naturally infected samples collected from cattle in Italy: Cooperia (52\%), Trichostrongylus (37\%), Ostertagia (7\%) and Haemonchus (4\%). 

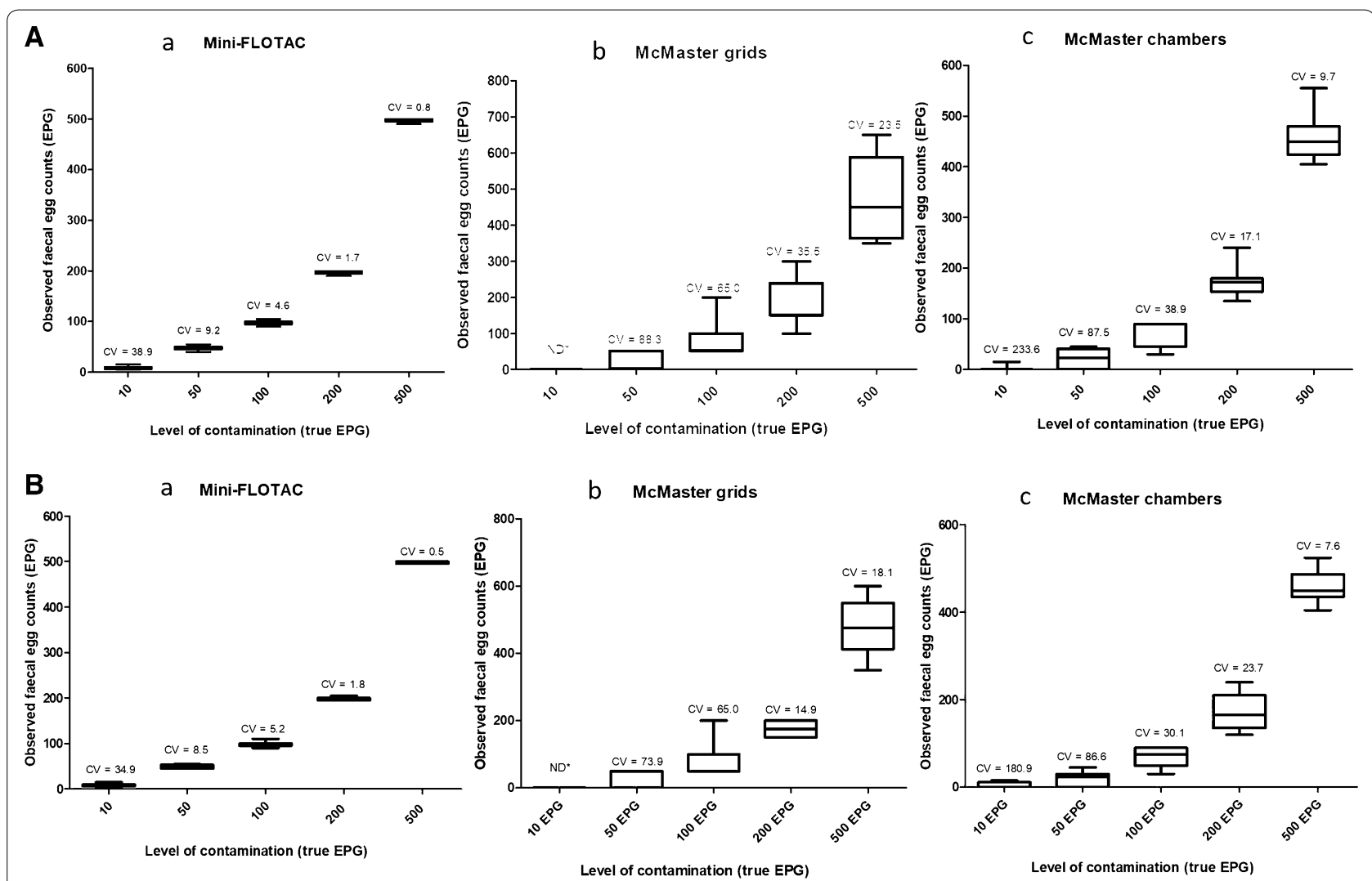

Fig. 1 Boxplots of observed faecal egg counts (y axis) with Mini-FLOTAC technique (a), McMaster grids (b) and McMaster chambers (c) for the five EPG levels of contamination in Belgium (A) and in Italy (B)

\section{Discussion}

The comparison between Mini-FLOTAC and McMaster for GIN FEC in cattle showed that Mini-FLOTAC had a higher sensitivity and accuracy and a lower CV than the McMaster technique (grids and chambers). Interestingly, McMaster grids showed higher FECs than McMaster chambers for all levels of contamination (10, 50, 100, 200 and 500 EPG). As described in Cringoli et al. [18] and Bosco et al. [12] it may be due to the tendency of eggs, during the flotation, to concentrate in the center of the McMaster slide, with a consequent overestimation of EPGs, especially at low egg counts. Moreover, McMaster showed no statistically significant difference between observed and true EPG only at 500 EPG and at grids level of reading. These results, therefore, showed that the McMaster is not a satisfactory method at low EPG levels, especially when the FECRT is used to evaluate the efficacy of anthelmintics and to detect anthelmintic resistance $[7,12,19]$.

In this study, the mean percentage of recovery of GIN eggs with Mini-FLOTAC was very high, i.e. 98.1\%. This result is in agreement with Godber et al. [10] and Bosco et al. [12] who found a $100 \%$ recovery rate of GIN eggs in
Table 1 Mean accuracy (\%) of Mini-FLOTAC and McMaster (grids and chambers) at the different EPG levels resulting from the experiment performed in Belgium and in Italy

\begin{tabular}{llllll}
\hline FEC method & 10 EPG & 50 EPG & 100 EPG & 200 EPG & 500 EPG \\
\hline Belgium & & & & & \\
Mini-FLOTAC & 95.8 & 96.7 & 97.9 & 98.5 & 99.4 \\
McMaster grids & 0 & 58.3 & 87.5 & 87.5 & 95.0 \\
McMaster chambers & 25.0 & 42.5 & 68.8 & 88.2 & 92.0 \\
Italy & & & & & \\
Mini-FLOTAC & 95.8 & 98.3 & 99.2 & 99.6 & 99.5 \\
McMaster grids & 0 & 66.7 & 87.5 & 87.5 & 95.8 \\
McMaster chambers & 37.5 & 40.0 & 68.8 & 84.4 & 91.3 \\
\hline
\end{tabular}

sheep spiked faeces. The study by Paras et al. [7] showed a $70.9 \%$ recovery rate of cattle GIN eggs by Mini-FLOTAC that was higher than the values by other techniques ( $30.9 \%$ by modified Wisconsin and $55.0 \%$ by McMaster), but lower than the value detected in our study (98.1\%). Similarly, Noel et al. [20] found a $42.6 \%$ recovery rate of equine strongyle eggs by Mini-FLOTAC, that was higher than the value from the McMaster technique (23.5\%). In 


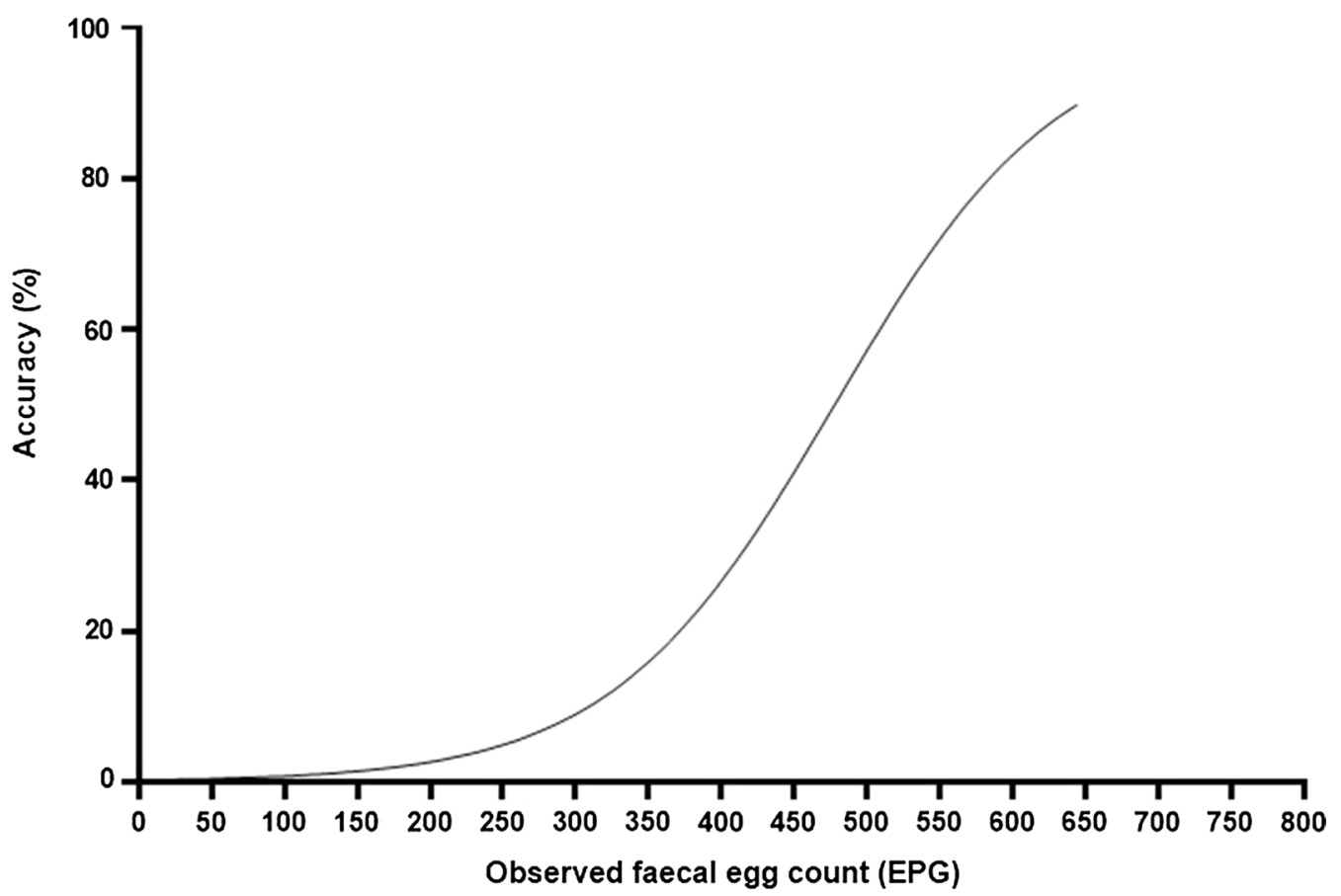

Fig. 2 The predicted accuracy derived from the logistic regression for McMaster technique at the grid level

the study on equine faecal samples by Napravnikova et al. [21] the accuracy of Mini-FLOTAC was 74.2\% (lower than McMaster) for strongyles and 90.3\% (higher than McMaster) for ascarids. Finally, Scare et al. [22] compared an automated FEC using a smartphone with MiniFLOTAC and McMaster and found a higher accuracy by Mini-FLOTAC (64.5\%) compared to McMaster (21.7\%) and the smartphone system (32.5\%).

As described in Cringoli et al. [8] and in Norris et al. [23], the procedure of egg isolation and faeces contamination, as well as the choice of the flotation solution may influence the recovery rates of a technique in any egg-spiking experiment. These factors may have contributed to the high recovery rate of GIN eggs in cattle using Mini-FLOTAC in the present study as argued below. First, with regard to the spiking procedure, in our study we spiked cattle faeces with GIN eggs obtained from catthe that were either experimentally (in Belgium) or naturally (in Italy) infected with GINs. This could explain the higher accuracy compared to the findings by Paras et al. [7] where eggs isolated from goat faeces were used to contaminate cattle faeces. In support of our hypothesis, a recovery rate only of $91.0 \%$ was obtained by Bosco et al. [12] when GIN eggs from sheep were added to horse faeces. Secondly, the choice of the flotation solution is very important, as it may influence the performance of the technique and therefore its precision and accuracy [8].
In different studies it has been shown that sodium chloride (specific gravity $=1.20$ ) was the best flotation solution for GIN FEC and it is recommended when using the Mini-FLOTAC technique [8]. Therefore, the low recovery rates found in the above mentioned studies could be due to the inappropriateness of the flotation solutions (i.e. sodium nitrate with a specific gravity $=1.25-1.30$ [7]; and glucose- $\mathrm{NaCl}$ flotation medium with a specific gravity $=1.24-1.28[20-22]$.

In our study, CVs of Mini-FLOTAC were lower than the CVs of McMaster grids and chambers for all levels of contamination as reported also in other studies [7, 10, 12, 13, 20-22, 24]. Furthermore, CVs for McMaster chambers were lower than those obtained with McMaster grids, in agreement with Godber et al. [10] and Bosco et al. [12]. To support these findings, Levecke et al. [25] and Torgerson et al. [26] showed that precision increases when analytical sensitivity increases; with the McMaster technique, the variance of EPG estimates between repeated samples of the same faecal sample is inflated, due to the multiplication factor when transforming the raw counts in EPG [26]. Moreover, in the present study CVs were lower at higher levels of contamination for all the techniques, as also reported by Mes et al. [27] and Das et al. [28], the precision increases when the EPG in faecal sample increases. 


\section{Conclusions}

Since the sensitivity, precision and accuracy of a FEC depend on many factors, it is very important to establish precise standard operating procedures (SOPs) for FEC techniques, including the flotation solution to use. In fact, it is surprising that diagnostic and research laboratories around the world use different protocols of FEC techniques for their activities. In this regard, research priorities should include the development of more scalable, reliable, less labour-intensive systems for parasite egg counts for both pen-side and laboratory use [5], including methods of automated sample processing and image analysis [29] as indicated in the STAR-IDAZ (https://www.star-idaz.net) diagnostic road map for research on helminths and anthelmintic resistance.

\section{Abbreviations}

AR: anthelmintic resistance; CV: coefficient of variation; EPG: egg per gram of faeces; FEC: faecal egg count; FECRT: faecal egg count reduction test; GIN: gastrointestinal nematode; L3: third-stage larvae; SOPs: standard operating procedures.

\section{Acknowledgements \\ The authors would like to express sincere appreciation to Nathalie De Wilde and Stijn Casaert (Department of Virology, Parasitology and Immunology, Faculty of Veterinary Medicine, Ghent University, Belgium) for their technical collaboration. This article is based upon work from COST Action COMBAR CA16230, supported by COST (European Cooperation in Science and Technology).}

\section{Authors' contributions}

LR, GC, EC and MPM conceived, designed and coordinated the study. AA and $A B$ performed sampling and laboratory analyses. All authors contributed to data analysis and preparation of the manuscript. All authors read and approved the final manuscript.

\section{Funding}

This research received no external funding.

\section{Availability of data and materials}

All data generated or analysed during this study are included in this published article. The datasets used and/or analysed during the present study available from the corresponding author upon reasonable request.

\section{Ethics approval and consent to participate}

We obtained verbal informed consent from the owners of animals to collect the faecal samples.

\section{Consent for publication}

Not applicable.

\section{Competing interests}

The Mini-FLOTAC technique was developed and is patented by GC, but the patent has been handed over to the University of Naples Federico II. GC is the current patent holder of the Mini-FLOTAC and Fill-FLOTAC and had no role in the preparation and submission of the protocols reported or the design and implementation of ongoing and future studies. To obtain Mini-FLOTAC or Fill-FLOTAC devices, a contribution is required that is used only to cover costs of production and packaging, and to contribute to the ongoing FLOTAC research. The remaining authors (AA, $A B, L R, E C$ and $M P M)$ declare that they have no competing interests.

\section{Author details}

${ }^{1}$ Department of Veterinary Medicine and Animal Production, University of Naples Federico II, CREMOPAR, Naples, Italy. ${ }^{2}$ Department of Virology, Parasitology and Immunology, Faculty of Veterinary Medicine, Ghent University, Ghent, Belgium.

Received: 16 January 2020 Accepted: 27 April 2020

Published online: 06 May 2020

\section{References}

1. Charlier J, Höglund J, von Samson-Himmelstjerna G, Dorny P, Vercruysse J. Gastrointestinal nematode infections in adult dairy cattle: impact on production, diagnosis and control. Vet Parasitol. 2009;164:70-9.

2. Sutherland IA, Leathwick DM. Anthelmintic resistance in nematode parasites of cattle: a global issue? Trends Parasitol. 2011;27:176-81.

3. Rose H, Rinaldi L, Bosco A, Mavrot F, de Waal T, Skuce P, et al. Widespread anthelmintic resistance in European farmed ruminants: a systematic review. Vet Rec. 2015:176:546.

4. Geurden T, Chartier C, Fanke J, di Regalbono AF, Traversa D, von SamsonHimmelstjerna $\mathrm{G}$, et al. Anthelmintic resistance to ivermectin and moxidectin in gastrointestinal nematodes of cattle in Europe. Int J Parasitol Drugs Drug Resist. 2015:5:163-71.

5. Rinaldi L, Amadesi A, Dufourd E, Bosco A, Gadanho M, Lehebel A, et al. Rapid assessment of faecal egg count and faecal egg count reduction through composite sampling in cattle. Parasit Vectors. 2019;12:353.

6. Vercruysse J, Charlier J, Van Dijk J, Morgan ER, Geary T, von Samson- Himmelstjerna $\mathrm{G}$, et al. Control of helminth ruminant infections by 2030. Parasitology. 2018;145:1655-64.

7. Paras KL, George MM, Vidyashankar AN, Kaplan RM. Comparison of fecal egg counting methods in four livestock species. Vet Parasitol. 2018;257:21-7.

8. Cringoli G, Maurelli MP, Levecke B, Bosco A, Vercruysse J, Utzinger J, et al. The Mini-FLOTAC technique for the diagnosis of helminth and protozoan infections in humans and animals. Nat Protoc. 2017;12:1723-32.

9. Rinaldi L, Levecke B, Bosco A, lanniello D, Pepe P, Charlier J, et al. Comparison of individual and pooled faecal samples in sheep for the assessment of gastrointestinal strongyle infection intensity and anthelmintic drug efficacy using McMaster and Mini-FLOTAC. Vet Parasitol. 2014;205:216-23.

10. Godber OF, Phythian CJ, Bosco A, lanniello D, Coles G, Rinaldi L, et al. A comparison of the FECPAK and Mini-FLOTAC faecal egg counting techniques. Vet Parasitol. 2015;207:342-5.

11. Kenyon F, Rinaldi L, McBean D, Pepe P, Bosco A, Melville L, et al. Pooling sheep faecal samples for the assessment of anthelmintic drug efficacy using McMaster and Mini-FLOTAC in gastrointestinal strongyle and Nematodirus infection. Vet Parasitol. 2016;225:53-60.

12. Bosco A, Maurelli MP, lanniello D, Morgoglione ME, Amadesi A, Coles GC, et al. The recovery of added nematode eggs from horse and sheep faeces by three methods. BMC Vet Res. 2018;14:7.

13. Dias de Castro LL, Abrahao CLH, Buzatti A, Molento MB, Bastianetto E, Rodrigues DS, et al. Comparison of McMaster and mini-FLOTAC fecal egg counting techniques in cattle and horses. Vet Parasitol Reg Stud Rep. 2017; 10:132-5.

14. George MM, Paras KL, Howell SB, Kaplan RM. Utilization of composite fecal samples for detection of anthelmintic resistance in gastrointestinal nematodes of cattle. Vet Parasitol. 2017:240:24-9.

15. Cringoli G, Rinaldi L, Maurelli MP, Utzinger J. FLOTAC: new multivalent techniques for qualitative and quantitative copromicroscopic diagnosis of parasites in animals and humans. Nat Protoc. 2010;5:503-15.

16. MAFF. Manual of Veterinary Parasitological Techniques. 3rd ed. London: Her Majesty's Stationary Office; 1986. p. 160.

17. van Wyk JA, Mayhew E. Morphological identification of parasitic nematode infective larvae of small ruminants and cattle: a practical lab guide. Onderstepoort J Vet Res. 2013;80:539.

18. Cringoli G, Rinaldi L, Veneziano V, Capelli G, Scala A. The influence of flotation solution, sample dilution and the choice of McMaster slide area (volume) on the reliability of the McMaster technique in estimating the faecal egg counts of gastrointestinal strongyles and Dicrocoelium dendriticum in sheep. Vet Parasitol. 2004;123:121-31. 
19. Van den Putte N, Claerebout E, Levecke B. Evaluation of the mini-FLOTAC technique for detection of gastro-intestinal parasites in large companion animals. Vlaams Diergeneeskunding Tijdschrift. 2016;85:15-22.

20. Noel ML, Scare JA, Bellaw JL, Nielsen MK. Accuracy and precision of MiniFLOTAC and McMaster techniques for determining equine strongyle egg counts. J Equine Vet Sci. 2017;48:182-7.

21. Nápravníková J, Petrtýl M, Stupka R, Vadlejch J. Reliability of three common fecal egg counting techniques for detecting strongylid and ascarid infections in horses. Vet Parasitol. 2019;272:53-7.

22. Scare JA, Slusarewicz P, Noel ML, Wielgus KM, Nielsen MK. Evaluation of accuracy and precision of a smartphone based automated parasite egg counting system in comparison to the McMaster and Mini-FLOTAC methods. Vet Parasitol. 2017;247:85-92.

23. Norris JK, Slusarewicz P, Nielsen MK. Pixel by pixel: real-time observation and quantification of passive flotation speeds of three common equine endoparasite egg types. Int J Parasitol. 2019;49:885-92.

24. Went HA, Scare JA, Steuer AE, Nielsen MK. Effects of homogenizing methods on accuracy and precision of equine strongylid egg counts. Vet Parasitol. 2018;261:91-5

25. Levecke B, Rinaldi L, Charlier J, Maurelli MP, Morgoglione ME, Vercruysse J, et al. Monitoring drug efficacy against gastrointestinal nematodes when faecal egg counts are low: do the analytic sensitivity and the formula matter? Parasitol Res. 2011;109:953-7.

26. Torgerson PR, Paul M, Lewis Fl. The contribution of simple random sampling to observed variations in faecal egg counts. Vet Parasitol. 2012;188:397-401.

27. Mes TH. Technical variability and required sample size of helminth egg isolation procedures. Vet Parasitol. 2003;115:311-20.

28. Das G, Savas T, Kaufmanna F, Idris A, Abela H, Gaulya M. Precision, repeatability and representative ability of faecal egg counts in Heterakis gallinarum infected chickens. Vet Parasitol. 2011;183:87-94.

29. Slusarewicz P, Pagano S, Mills C, Popa G, Chow KM, Mendenhall M, et al. Automated parasite faecal egg counting using fluorescence labelling, smartphone image capture and computational image analysis. Int J Parasitol. 2016:46:485-93.

\section{Publisher's Note}

Springer Nature remains neutral with regard to jurisdictional claims in published maps and institutional affiliations.
Ready to submit your research? Choose BMC and benefit from:

- fast, convenient online submission

- thorough peer review by experienced researchers in your field

- rapid publication on acceptance

- support for research data, including large and complex data types

- gold Open Access which fosters wider collaboration and increased citations

- maximum visibility for your research: over $100 \mathrm{M}$ website views per year

At BMC, research is always in progress.

Learn more biomedcentral.com/submissions 